\title{
Factores que inciden en el fomento del programa de ingeniería de sistemas. Una perspectiva de la comunidad estudiantil
}

\section{Factors that affect the promotion of the systems engineering program. A perspective of the student community}

Msc. Mario Samuel Rodríguez Barrero

Universidad Cooperativa de Colombia, Colombia

PhD Gustavo Adolfo Rubio Rodríguez

Universidad Cooperativa de Colombia, Colombia

PhD Mario Heimer Flórez Guzmán

Corporación Universitaria Remington, Colombia

PhD Nubia Varón Triana

Universidad Cooperativa de Colombia, Colombia

Autor para correspondencia: mario.rodriguezb@campusucc.edu.co, gustavo.rubio@campusucc.edu.co,mariohfg@hotmail.com,nubia.varont@campusucc.edu.co Fecha de recepción: 24 de Mayo de 2017 - Fecha de aceptación: 15 de Septiembre de 2017

Resumen: El presente artículo, caracteriza el programa académico de ingeniería de sistemas que actualmente ofertan las instituciones de educación superior en el departamento del Tolima, Colombia. Esta investigación es de tipo cuantitativo con enfoque explicativo, y estableció una muestra de 400 estudiantes de último grado de bachillerato, de 6 establecimientos educativos (privados y oficiales), y 141 estudiantes de los primeros semestres del programa de ingeniería de sistemas de las principales universidades del departamento antes mencionado. Como resultado del análisis de la información obtenida, se determinó que las universidades de la región deben formular un plan de marketing, el cual promueva un análisis situacional que genere estrategias que permitan hacer más competitivo el programa de ingeniería objeto de estudio.

Palabras Clave: programa de ingeniería de sistemas; posicionamiento; marketing educativo

\begin{abstract}
This article characterizes the academic systems engineering program currently offered by higher education institutions in the department of Tolima, Colombia. This research is quantitative with explanatory focus, and established a sample of 400 students of the last grade of baccalaureate, of 6 educational establishments (private and official), and 141 students of the first semesters of the systems engineering program of the main universities of the aforementioned department. As a result of the analysis of the information obtained, it was determined that universities in the region should formulate a marketing plan, which promotes a situational analysis that generates strategies that make the engineering program object of study more competitive.
\end{abstract}

Key words: program of systems engineering; positioning; educational marketing 


\section{Introducción}

El programa de ingeniería de sistemas es de gran importancia para el desarrollo tecnológico de los países en vía de desarrollo, por esta razón en Colombia se ha creado el Ministerio de las TICS, el cual fomenta la educación en el uso de las nuevas tecnologías, pues se considera que la productividad y competitividad del país depende en gran parte del buen uso de las herramientas tecnológicas; asimismo, la innovación tecnológica fuente de desarrollo, depende de la formación en los sistemas de información, por lo cual la demanda de programas como el de ingeniería de sistemas es un tema de interés nacional que se aborda incluso en los planes de desarrollo nacional, regional y local.

Por lo anteriormente expuesto, a través de esta investigación se pretende analizar las características que ostentan los programas de ingeniería de sistemas de las universidades del departamento del Tolima que brindan esta carrera académica, para lo cual, inicialmente se determinaron los factores que inciden en la elección del programa por parte de los estudiantes de ingeniería de sistemas de las diferentes universidades del departamento, luego se analizaron estos factores, se definió el perfil de los aspirantes al programa de ingeniería de sistemas, y finalmente se plantearon una serie de recomendaciones con el propósito de posicionar este programa e incrementar su demanda.

\section{Posicionamiento}

\section{Marco teórico}

En primer lugar, se define el posicionamiento como la posición de un producto y forma como los consumidores lo definen, de acuerdo con atributos importantes. Es el lugar que el producto ocupa en la mente del consumidor, en relación con los otros productos de la competencia (Philip \& Armstrog, 2003), es decir, que para definir el posicionamiento del producto no solo se deben observar sus atributos, sino, su relación con sus competidores y productos sustitutos, pues es la percepción de los clientes, consumidores o usuarios, mediante la cual se puede definir el posicionamiento y no desde la perspectiva interna de la empresa. En este contexto, se puede decir que las estrategias de posicionamiento crean una protección para las ventas en el mercado, pues el posicionamiento no sólo nos ayuda a mantener un liderazgo, sino, que también es una herramienta útil para mantener una posición, evitar una colisión directa con el líder del mercado, introducir nuevos productos, ayudar a la diferenciación e identificación de productos en mercados saturados y prevenir los efectos de futuros lanzamientos o nuevas tecnologías (Marrón , 2014).

En el caso particular del programa de ingeniería de sistemas ofertado por las universidades que operan en la región del Tolima, se puede acotar que su posicionamiento guarda relación con la percepción que presentan sus grupos de interés (estudiantes de bachillerato, padres de familia, comunidad en general, comunidad universitaria, docentes, administrativos, gobierno), y por supuesto la competencia, conformada por los programas de ingeniería de sistemas y afines que se ofertan en dicha región. Su posición en el mercado dependerá entonces de la percepción que los interesados, estudiantes, egresados y demás stakeholders posean de este programa. Para lograr el mejor posicionamiento, se deben generar estrategias que le permitan visibilizarse como un programa de excelente calidad que proyecte a sus egresados en el mercado laboral. 


\section{Estrategia de posicionamiento}

Determinado el segmento objetivo, la empresa ha de establecer una estrategia para posicionar firmemente su producto en el segmento elegido. Para ello, hay que considerar tres aspectos esenciales, según Chaquilla (2013):

- El análisis del entorno y la competencia

- El estudio de las necesidades del consumidor

- La evaluación de los puntos fuertes y débiles de la propia empresa

El análisis de tales factores permite determinar la oportunidad de diferenciar la marca ante el público.

\section{Marketing mix}

Philip Kotler define el marketing como un proceso social y gerencial (Philip \& Armstrog, 2003); en el aspecto social los individuos y grupos crean intercambios de productos y valores con otros, con la finalidad de obtener lo que necesitan y desean. En cuanto al aspecto gerencial, éste ha sido descrito como el proceso donde se da el "arte de vender productos", también se identifica de una manera más breve el marketing mix, como el proceso en donde son identificadas y satisfechas las necesidades humanas y sociales de una manera rentable. (Philip \& Armstrog, 2003). Para el logro de los diferentes objetivos planteados desde los diferentes puntos de vista, ya sea social o gerencial, se hace necesario el uso de diferentes herramientas conocidas como marketing mix o mezcla de mercadeo. McCarthy, quien a mediados del siglo XX lo denominó la teoría de las «cuatro pes», en razón a que utiliza cuatro variables, cuyas iniciales en inglés empiezan por «p» (Muñiz, 2014).

$\begin{array}{llll}\text { - } & \text { Product } & \rightarrow & \text { Producto } \\ \text { - } & \text { Place } & \rightarrow & \text { Distribución - Venta } \\ \text { - } & \text { Promotion } & \rightarrow & \text { Promoción } \\ \text { - } & \text { Price } & \rightarrow & \text { Precio }\end{array}$

En la siguiente tabla se pueden observar los diferentes elementos que conforman el marketing mix con sus respectivos conceptos.

Tabla 1. Variables del marketing mix

\begin{tabular}{lc}
\hline $\begin{array}{l}\text { Variable del } \\
\text { marketing mix }\end{array}$ & Concepto \\
\hline Precio & Se toma como la suma de los valores que los consumidores dan a cambio de los \\
beneficios de adquirir un bien o servicio & Es un conjunto de características tangibles e intangibles creadas con el fin de \\
producto & satisfacer una necesidad \\
& Comprende las actividades y el conjunto de elementos implícitos para la \\
Plaza & disposición de un producto de modo que sea adquirible para un consumidor \\
Promoción & Considera las actividades que comunican y que están enfocadas a convencer al \\
& cliente para la compra del producto \\
\hline
\end{tabular}

Fuente: Elaboración propia 
En la figura 1 se presentan también los elementos del marketing mix con cada uno de sus componentes.

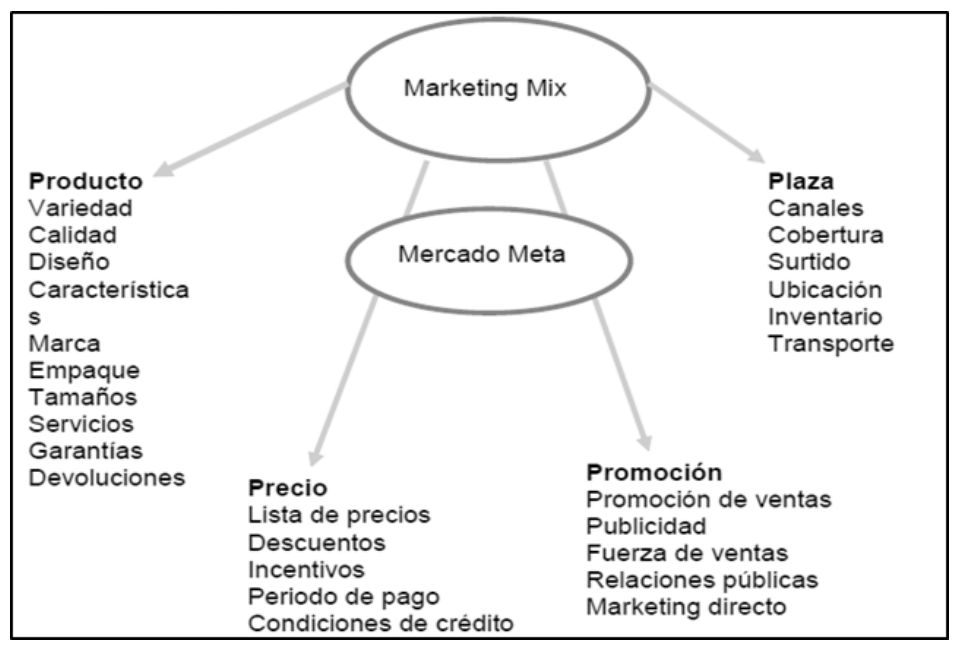

Figura 1. Herramientas del marketing mix

Fuente: Dirección de Marketing (Philip \& Armstrong, 2003, p.19)

Teniendo en cuenta la naturaleza del trabajo investigativo, se hace necesario tomar como modelo para la creación de estrategias de marketing la propuesta de Maciá Domene \& Gosende Grela.

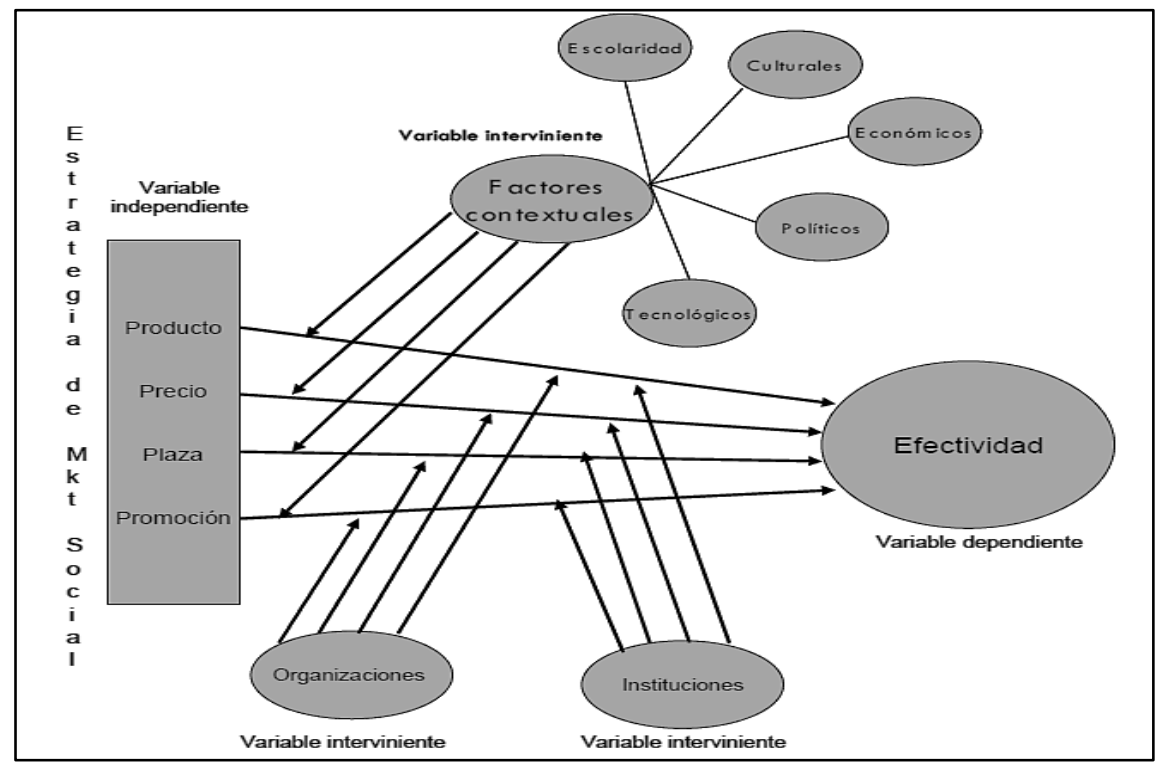

Figura 2. Modelo de marketing social

Fuente: Modelo adaptado de Maciá \& Gosende (2011)

\section{El marketing educativo}

Según una publicación realizada por Indemer "el marketing educativo es el orientador de la estrategia de las instituciones de educación, permitiendo analizar, planificar, ejecutar y 
controlar los procesos de intercambio entre los jóvenes (matricula potencial), la Institución, la empresa, la familia, la comunidad y otras escuelas", y es definido como el conjunto de actividades de mercadeo que realizan las instituciones educativas que propician el intercambio entre los diferentes componentes del entorno y de los estudiantes con la escuela. La mercadotecnia educacional se encarga de aportar la información necesaria para que los directivos de las instituciones de educación superior logren establecer un plan estratégico donde conjuguen los objetivos a alcanzar con los recursos disponibles, tanto materiales como humanos, dando respuestas de mayor calidad a las necesidades detectadas en los estudiantes y en el mercado laboral (INDEMER, 2010).

Las instituciones educativas pertenecen al sector de los servicios, lo cual hace que desde la óptica del marketing posean unas características que no sólo las diferencias de otras, sino, que son la base de su identidad. Desde luego, hay diferencias entre vender productos y prestar servicios de educación, pero ambos son intercambios de valor y no solamente expresados en términos monetarios, sino también el valor percibido por los individuos y el atribuido por los grupos sociales a los bienes y servicios (Saldaña, 1999). Zapata (2010), en el desarrollo de sus estudios, plantea que se debe hablar de "marketing educativo", para poder abordar de manera más sistemática la investigación de mercado y las posibilidades de cobertura con el fin de atender una demanda que requiere cada vez más servicios oportunos y personalizados.

Hoy en día se hace necesaria la implementación del marketing en las instituciones de educación, ya sean públicas o privadas, lo que se debe a que la universidad se asemeja a una organización de servicios en la que existe una relación de intercambio con los alumnos, sus familias, las empresas, la sociedad y otros actores ante los cuales debe responder como cualquier empresa ante las necesidades reales del mercado. Cuando se considera la conveniencia de aplicar los conceptos y técnicas de Marketing a la gestión de las Universidades, hay que considerar esas diferencias, implicando con ello la importancia de determinar los objetivos de la institución, lo cual dependerá de los fines que una Universidad determinada trate de lograr. Por ejemplo, la orientación al mercado y aunque no se aplique completamente a las Universidades, puede ayudar a conocer los cambios del entorno, para tenerlos en cuenta en el diseño de la oferta formativa (Naranjo, 2011). Finalmente, podemos decir que los servicios, incluyendo los educativos, presentan diferencias con respecto a los bienes, y que requieren de una estrategia de marketing apropiada para su exitosa administración.

\section{La demanda de los programas de ingeniería en Colombia}

Para el desarrollo del trabajo investigativo se tomará como base los aportes realizados por Salas (2002), quien clasifica los determinantes de las elecciones educativas en tres grandes categorías: los que están relacionados con el estudiante o los estudios que van a realizar, los determinantes que corresponden a características objetivas o subjetivas y los factores relacionados con las expectativas futuras como lo muestra la figura 3. 


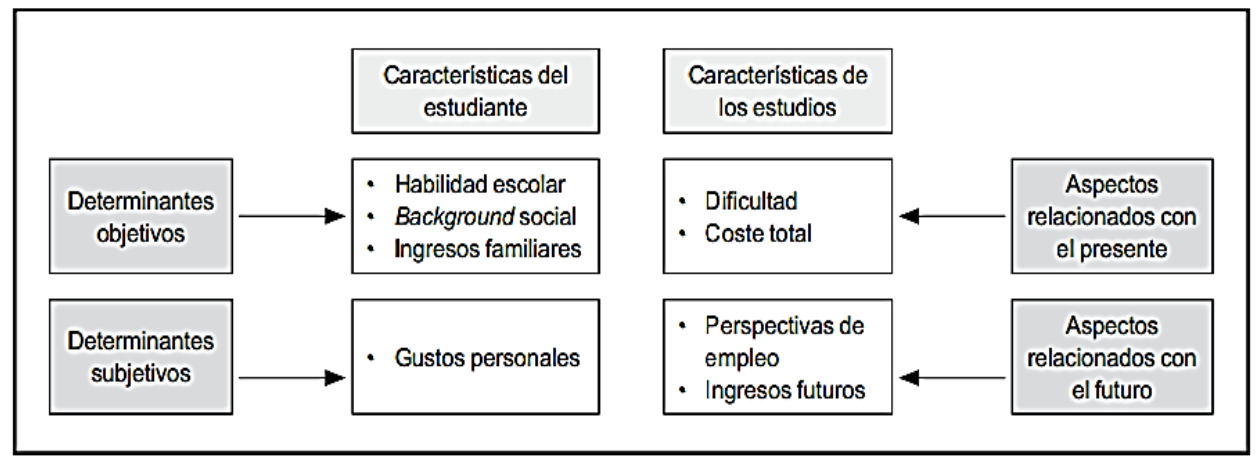

Figura 3. Determinante de las elecciones educativas en el ámbito universitario Fuente: (Salas, 2002)

Otros estudios relacionados con la educación y su demanda anotan que la educación en un bien normal y que su demanda depende principalmente de los ingresos de los familiares, por otro lado encuentra la relación de su función de utilidad presente o futura que es propia a cada individuo evaluando según criterio subjetivo que tiene más valor para ellos si la retribución económica o la satisfacción personal, pese a las diferencias subjetivas que puedan darse hay algunas supuestos que se pueden tomar en lo referente a la elección de carrera (Eicher, 1988) entre ellos están los siguientes :

- Los estudiantes prefieren una carrera que ofrecen unas tasas de rendimiento anticipado elevada

- Los estudiantes prefieren carreras en las que tienen oportunidad de éxito

- Generalmente las carreras que tienen estudios más difíciles ofrecen tasas más elevadas de rendimiento en donde debe darse el proceso de elección

- Los estudiantes con menores ingresos familiares dan más valor al proceso de elección.

\section{La ingeniería de sistemas}

\section{Historia de la ingeniería de sistemas en Colombia}

Las aplicaciones de la época en ingeniería de sistemas en Colombia, se limitaban al uso administrativo en los años 60s, ya que los sistemas operacionales y los lenguajes de programación eran totalmente restringidos.

En 1968 las universidades, Nacional, Andes e Industrial de Santander, establecen dentro de su oferta el programa de ingeniería de sistemas y computación. Los primeros profesionales de ingeniería de sistemas generados en las universidades colombianas hacia los años 1972-1973 eran contratados en su gran mayoría por las grandes empresas públicas y privadas, que contaban con computadoras (Universidad Cooperativa de Colombia, 2013)

\section{Plan de marketing}

Según la American Marketing Asociation (A.M.A.), el plan de marketing es un documento compuesto por un análisis de la situación de mercadotecnia actual, el análisis de las oportunidades y amenazas, los objetivos de mercadotecnia, la estrategia de mercadotecnia, los 
programas de acción y los ingresos proyectados (el estado proyectado de pérdidas y utilidades). Este plan puede ser la única declaración de la dirección estratégica de un negocio, pero es más probable que se aplique solamente a una marca de fábrica o a un producto específico. En última situación, el plan de marketing es un mecanismo de la puesta en práctica que se integra dentro de un plan de negocio estratégico total (Stanton, et al., 2013).

- Etapa de observación: Al igual que en el método científico y en otras metodologías de la investigación, el paso número uno es la observación, en este caso del entorno, el contexto y los factores que rodean. Es importante observar la audiencia, cómo se comporta en los medios sociales y las posibilidades que tendría la marca en estas plataformas.

- Etapa operativa: Aquí, ya con base en lo observado, se hace un planeamiento estratégico de las acciones a implantar.

- Etapa de ejecución: En esta fase se llevan a cabo las estrategias, es la puesta en marcha de cada una de las acciones establecidas, según los objetivos deseados.

- Etapa de evaluación y control: Al igual que toda acción es importante medir los resultados para evaluar si se está consiguiendo lo deseado, si no es momento de hacer cambios para mejorar y así alcanzar las metas (Rojas \& Maria , 2013)

\section{Diseño metodológico}

Como se mencionó anteriormente, el objetivo de este trabajo investigativo consiste en caracterizar el programa académico de ingeniería de sistemas, que en la actualidad ofertan las universidades del departamento del Tolima, Colombia. Para lograr este objetivo se adelantó una investigación de tipo cuantitativo con un enfoque explicativo (Hernández S, Fernández C, \& Baptista L, 2010), pues se pretende establecer las causas del fenómeno de estudio, que en este caso reside en identificar los factores que inciden en la promoción del programa de ingeniería de sistemas.

La metodología empleada se fundamenta entonces en una investigación de carácter cuantitativo, donde se aplicó el método inductivo, pues inicialmente se buscó establecer cuáles son las variables que incurren en la decisión de elección de una carrera universitaria por parte de los estudiantes de último grado de bachillerato. Luego, se identificaron los factores que incidieron en aquellos estudiantes que están cursando los primeros semestres de ingeniería de sistemas. Finalmente, se analizaron aquellas variables que influyen en la elección de carrera de ingeniería de sistemas ofertada por las 3 universidades representativas de la región.

\section{Población}

Para obtener la información primaria de esta investigación, se determinan a continuación las poblaciones objeto de estudio, en las cuales se observará el comportamiento de las variables del fenómeno a analizar. La primera población está conformada por los estudiantes del último grado de bachillerato de la ciudad de Ibagué, capital del departamento del Tolima; es decir, que esta población la constituyen los estudiantes que cursan el grado $11^{\circ}$ y que han presentado la prueba de ingreso a la educación superior Saber $11^{\circ}$. Según registro del SIMAT (Sistema de matrícula estudiantil), para el año en curso un total de 6028 estudiantes se encontraban matriculados y activos en colegios oficiales y privados según se muestra en la tabla 2. 
Tabla 2. Reporte de estudiantes de grado matriculados a grado 11 en la ciudad de Ibagué (semestre A de 2017)

\begin{tabular}{ll}
\hline Sectores & Jornada Ordinaria \\
\hline $\begin{array}{l}\text { Estudiantes } \\
\text { Sector no oficial }\end{array}$ & 1240 \\
$\begin{array}{l}\text { Estudiantes } \\
\text { Sector oficial } \\
\text { Total estudiantes }\end{array}$ & 4788 \\
\hline
\end{tabular}

Fuente: Elaboración propia

El segundo tipo de población está conformado por los estudiantes que cursan los primeros semestres del programa de ingeniería de sistemas de las 3 universidades distintivas del departamento del Tolima. Para este tipo de población, se consideraron estudiantes de los primeros semestres que se encontraban matriculados en el I, II y III semestre durante el período académico B de 2016. La tabla 3 así lo enseña:

Tabla 3. Universidades que ofertan el programa en Ibagué

\begin{tabular}{ll}
\hline Universidad & Modalidad \\
\hline Universidad Cooperativa de Colombia & Presencial \\
Universidad del Tolima & Distancia \\
Universidad de Ibagué & Presencial \\
UNAD & (Distancia - Virtual) \\
Corporación Universitaria CUN & Presencial \\
Universidad Antonio Nariño & Distancia \\
Fundación Universitaria San Martin & Distancia \\
\hline
\end{tabular}

Fuente: Elaboración propia

\section{Muestra}

Para la selección de los elementos muéstrales del primer tipo de población (estudiantes de grado $11^{\circ}$ ) se aplicó el método probabilístico, en el cual todas las unidades muestrales tuvieron la misma probabilidad de ser seleccionadas; para las otras poblaciones, se utilizó el método de muestreo no probabilístico por conveniencia o juicio del investigador, dado que no es posible seleccionar los elementos de forma aleatoria. El muestreo por conveniencia según Hernández (2004), consiste en seleccionar las unidades muéstrales más convenientes para el estudio o en permitir que la participación de la muestra sea totalmente voluntaria.

Para el segundo tipo de población se aplicó una encuesta a estudiantes utilizando el método de muestreo intencional; este tipo de muestreo se caracteriza por un esfuerzo deliberado de obtener muestras "representativas" mediante la inclusión en la muestra de grupos supuestamente típicos (Rojas Villarce, 2014). Para este trabajo investigativo se han elegido las tres universidades con mayor demanda de la ciudad, la universidad cooperativa de Colombia, la universidad del Tolima y la universidad de Ibagué.

\section{Análisis de resultados}




\section{Análisis encuestas a estudiantes de grado $11^{\circ}$}

Objetivo: Determinar los motivos de elección de carrera profesional de los estudiantes de último grado de bachillerato aspirantes a la educación superior en la ciudad de Ibagué.

Desarrollo: Se encuestaron un total de 400 estudiantes en las siguientes instituciones:
Colegios Públicos (202 estudiantes)
- INEM
- Instituto Técnico Rosarista
- Institución Educativa Alberto Castilla
- Exalumnas de la Presentación Instituciones privadas (198 estudiantes)
- Augusto E. Medina - Comfenalco
- Colegio Tolimense

El $57 \%$ de los estudiantes encuestados está conformado por mujeres y el $43 \%$ hombres, como se muestran en la figura numero 4; la carrera con mayor demanda es medicina, seguida de psicología, derecho, ingeniería industrial y negocios internacionales en su respectivo orden.

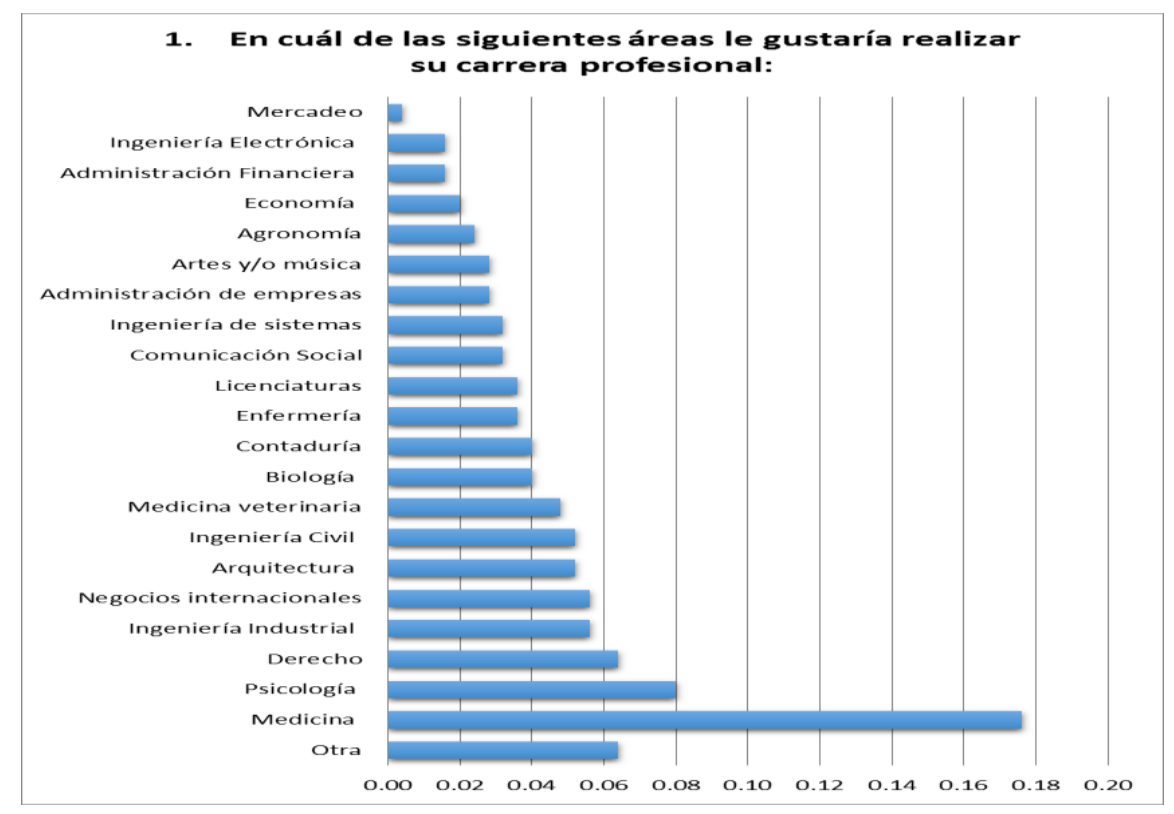

Figura 4. Tendencias de elección de carrera

Fuente: Elaboración propia

Haciendo el análisis de los motivos más relevantes de los estudiantes de grado once en el momento de elegir su carrera universitaria, se evidencia que más del $90 \%$ de los estudiantes eligen la carrera universitaria por gusto agrado o afinidad, seguido por los motivos de habilidades escolares y la demanda laboral; es decir, que lo que más les motiva en el momento de elección de carrera son factores personales como lo describe Salas (2002), e igualmente atienden al factor social de demanda laboral. 


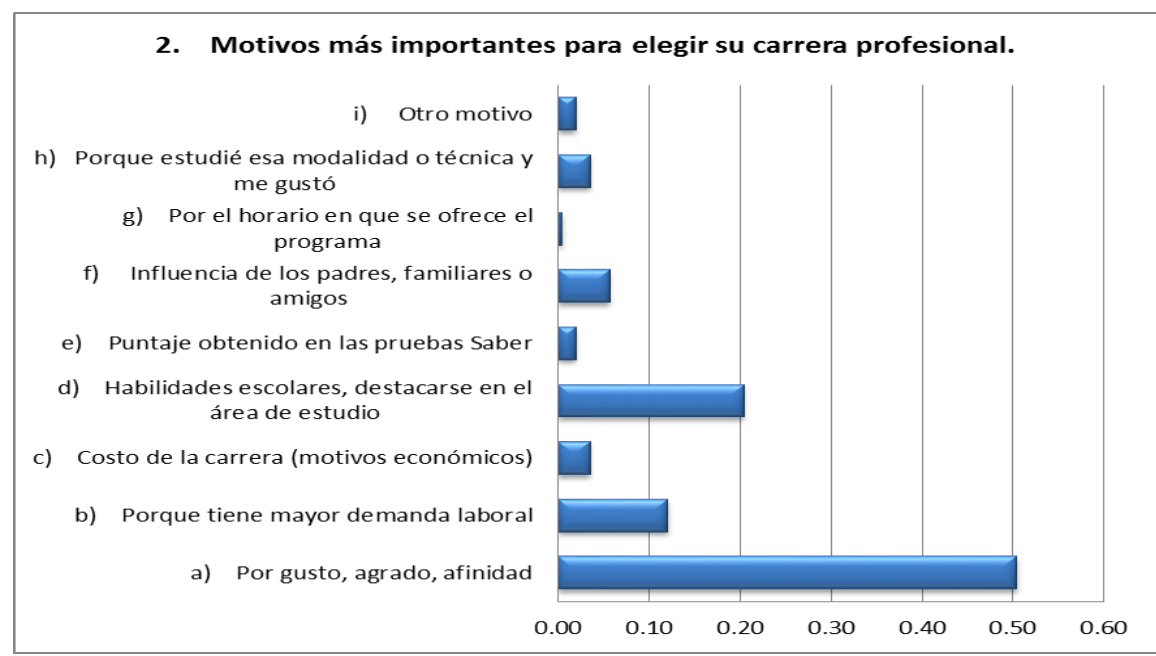

Figura 5. Motivos más importantes para la elección de carrera

Fuente: Elaboración propia

De los 16 estudiantes que eligieron ingeniería de sistemas como posible carrera a estudiar, las universidades con mayor proyección de demanda fueron la Universidad de Ibagué con 7 estudiantes, la Universidad del Tolima con 6 estudiantes y 3 de la Universidad Cooperativa de Colombia. Los estudiantes eligen la Universidad de Ibagué, y el motivo con mayor frecuencia de respuesta por el cual elegiría esta universidad es porque oferta la carrera.

\begin{tabular}{|c|c|c|c|c|c|c|c|c|c|c|c|c|c|c|}
\hline & sigu & i & e & ive & sidades le gustaría cursa & :a & ese & Un & iversi & idad & par &.- & iz & su \\
\hline 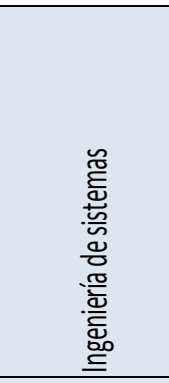 & 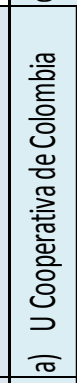 & 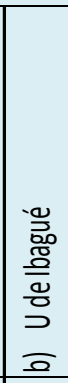 & 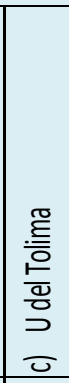 & 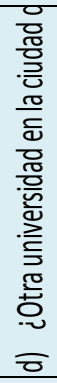 & 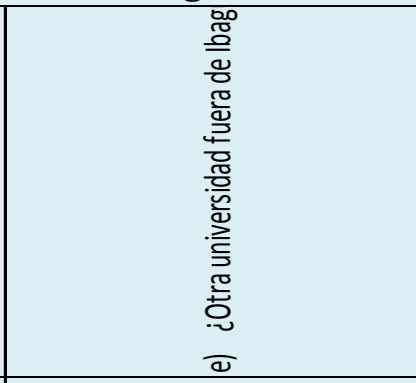 & 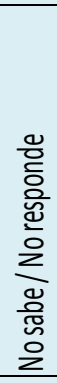 & 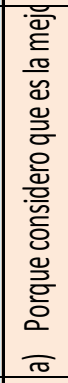 & 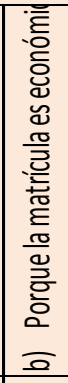 & 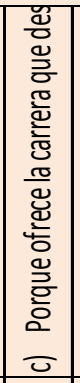 & 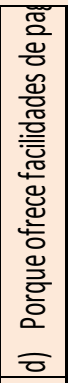 & 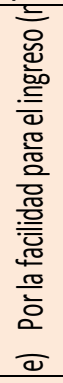 & 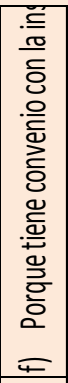 & 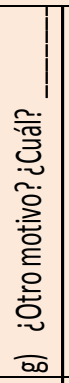 & 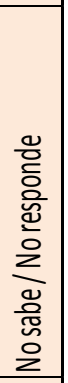 \\
\hline$x$ & $x$ & & & & U. Antioquia & & $x$ & & & & & & & \\
\hline$x$ & & $x$ & & & & & & $x$ & & & & & & \\
\hline$x$ & & & $x$ & & & & $x$ & & & & & & & \\
\hline$x$ & & & $x$ & & & & $x$ & & & & & & & \\
\hline$x$ & & & $x$ & & & & & & $x$ & & & & & \\
\hline$x$ & & $x$ & & & UNIVERSIDAD NACIONAL & & & $\mathrm{x}$ & & & & & & \\
\hline$x$ & & $x$ & & & & & & $x$ & & & & & & \\
\hline$x$ & & $x$ & & & & & & & & & & & & \\
\hline$x$ & & $x$ & & & & & & $x$ & & & & & & \\
\hline$x$ & & & $x$ & & & & $x$ & & & & & & & \\
\hline$x$ & & & $x$ & & & & $x$ & & & & & & & \\
\hline$x$ & & $x$ & & & & & & $x$ & & & & & & \\
\hline$x$ & & $x$ & & & & & & $x$ & & & & & & \\
\hline$x$ & & & $x$ & & & & $x$ & & & & & & & \\
\hline$x$ & $x$ & & & & & & & $x$ & & & & & & \\
\hline$x$ & $x$ & & & & & & & & & $x$ & & & & \\
\hline TOTAL 16 & 3 & 7 & 6 & & & & 6 & 7 & 1 & 1 & & & & \\
\hline
\end{tabular}

Figura 6. Análisis de la relación entre ingeniera de sistemas, universidad y motivos más importantes para la elección de carrera

Fuente: Elaboración propia 


\section{Análisis encuestas a estudiantes de primeros semestres de ingeniera de sistemas}

Objetivo: Identificar los motivos por los cuales los estudiantes de ingeniería de sistemas eligieron esa carrera.

Desarrollo: Con el fin de recolectar información primaria y determinar los motivos por los cuales los estudiantes eligieron la carrera de ingeniería de sistemas, se encuestaron 141 estudiantes de 3 universidades donde se oferta esta carrera: Universidad Cooperativa de Colombia, Universidad del Tolima y Universidad de Ibagué. El instrumento de recolección de información contempla preguntas relacionadas con el género, estrato, los motivos de elección de carrera y de universidad, los medios que utilizaron para buscar información sobre la carrera, las habilidades, actitudes de los estudiantes del programa y finalmente se abordaron preguntas relacionadas con el pensum, la metodología de enseñanza, la infraestructura física y tecnológica y el precio de la matrícula, información que será relevante para determinar el perfil de los aspirantes al programa de ingeniería de sistemas y diseñar estrategias pertinentes para la promoción del mismo.

En primer lugar, se identificó que el $80 \%$ de los encuestados son hombres, demostrando que la gran mayoría de los aspirantes y estudiantes de ingeniería de sistemas pertenecen al género masculino, y solo una quinta parte de los estudiantes encuestados son mujeres. El análisis de los estratos nos señala que el $48 \%$ de la población encuestada pertenece al estrato 2, el estrato 1 representa el 15\%, el estrato 3 el $25 \%$ y el estrato 4 el $11 \%$. En este aspecto cabe acotar que la estratificación social varía notablemente en cada institución, dado que ocupan diferentes nichos de mercado como se puede observar en la tabla número 4.

Tabla 4. Estratos de los estudiantes encuestados por universidad

\begin{tabular}{|c|c|c|c|c|c|c|}
\hline Universidad & 1 & 2 & 3 & 4 & 5 & 6 \\
\hline Universidad del Tolima & $24 \%$ & $65 \%$ & $10 \%$ & $2 \%$ & $0 \%$ & $0 \%$ \\
\hline Universidad Coop & $9 \%$ & $40 \%$ & $32 \%$ & $18 \%$ & $0 \%$ & $2 \%$ \\
\hline Universidad de Ibagué & $5 \%$ & $19 \%$ & all $52 \%$ & $24 \%$ & $0 \%$ & $0 \%$ \\
\hline
\end{tabular}

Fuente: Elaboración propia

Teniendo en cuenta esta información, se puede observar que el $89 \%$ de los estudiantes encuestados de la universidad del Tolima pertenecen a los estratos 1 y 2 , lo cual obedece a que es la única universidad pública de la región. Asimismo, se observa que el $90 \%$ de los estudiantes de la universidad cooperativa de Colombia pertenecen a los estratos 2,3 y 4, mientras que esta tendencia se observa en un $95 \%$ en la universidad de Ibagué, en la cual se observa que más del $50 \%$ de sus estudiantes pertenece al estrato 3 . 


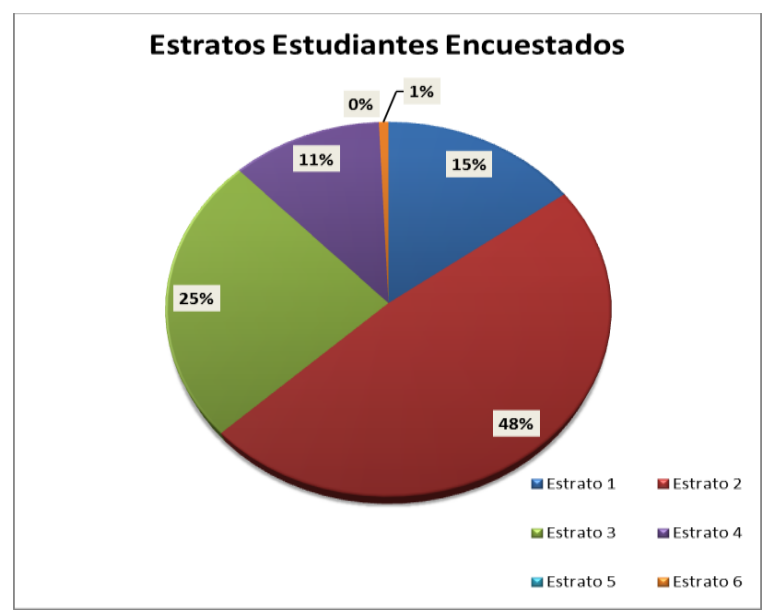

Figura 7. Estrato estudiantes encuestados

Fuente. Elaboración propia

Como parte de los motivos más importantes para elegir su carrera profesional, los estudiantes tuvieron en cuenta su gusto o grado de afinidad en un 59\%, un $19 \%$ por haber estudiado esta modalidad, un $8 \%$ por tener mayor demanda laboral al igual que por el horario. Los resultados detallados se muestran en la figura número 8.

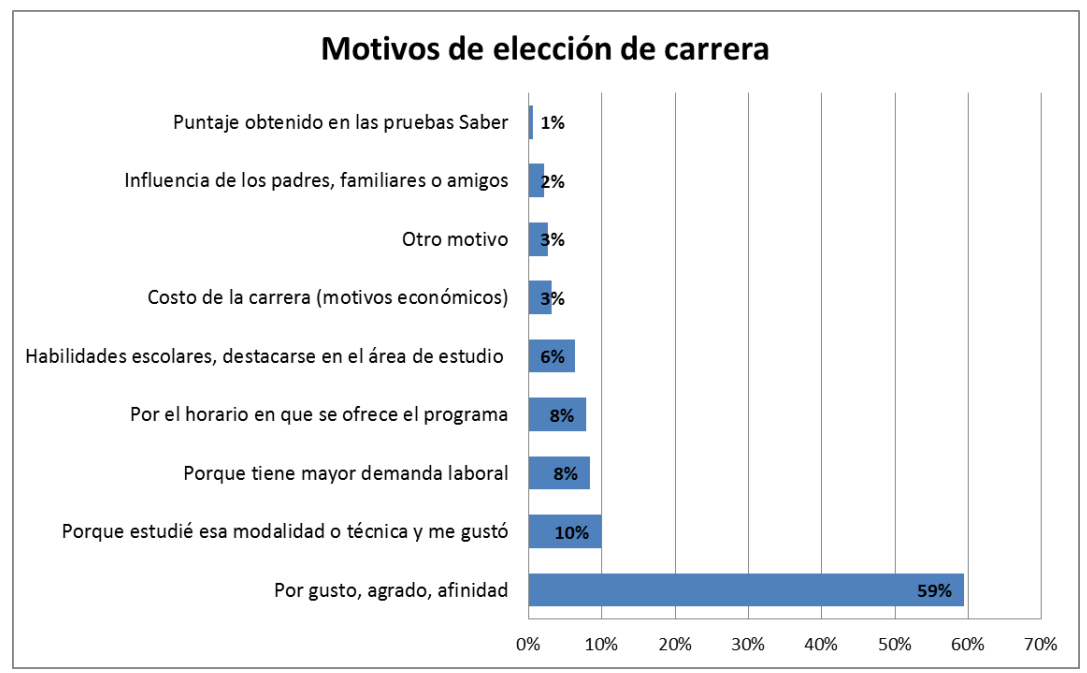

Figura 8. Motivos de elección de carrea

Fuente. Elaboración propia

Los resultados obtenidos revelan que los medios por los cuales los encuestados se informaron sobre su carrera profesional, fueron la página de la universidad con el $49 \%$ de favorabilidad, seguido de la solicitud de información directa con un $28 \%$, como se enseña en la figura número 9. 


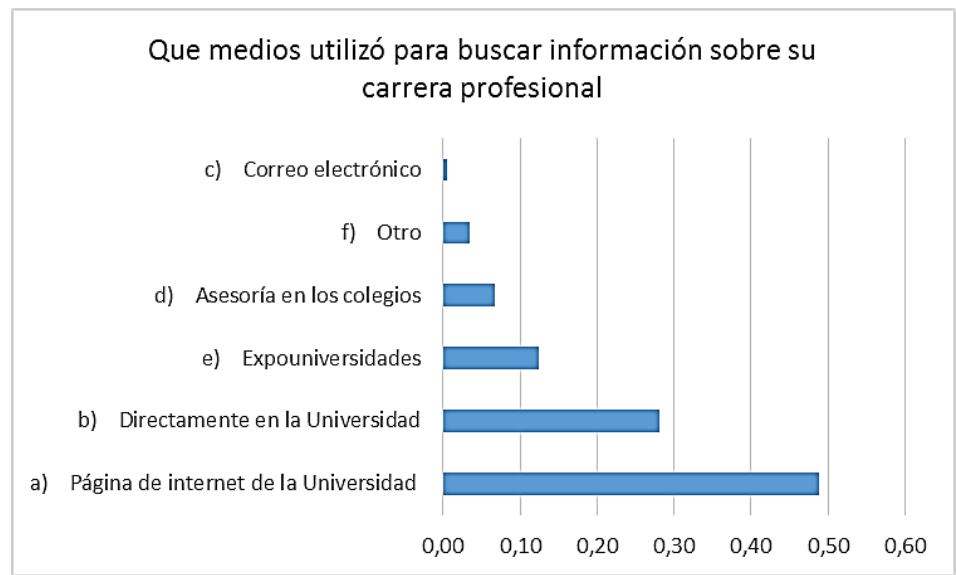

Figura 9. Medios para buscar información sobre su carrera Fuente: Elaboración propia

Al consultar los motivos por los cuales los encuestados eligieron la universidad para realizar sus estudios, se encontró que el 35\% considera la institución seleccionada como la mejor institución, un $24 \%$ tuvo en cuenta que era la universidad en la cual se ofertaba la carrera, y un porcentaje igual por motivos económicos. Los resultados de dicha pregunta se presentan en la figura número 10 .

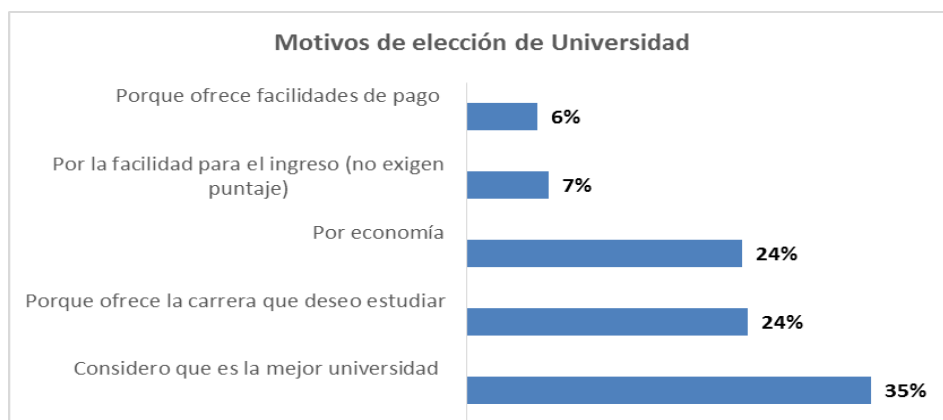

Figura 10. Motivo de elección de universidad Fuente: Elaboración propia

Con relación a las habilidades necesarias para estudiar la carrera, los encuestados consideran que para estudiar ingeniería de sistemas se requiere capacidad de análisis crítico y razonamiento lógico y abstracto un $28 \%$, capacidad creatividad y gusto por la lectura un 16 y $15 \%$, respectivamente. Igualmente, consideran importante tener pensamiento analítico y sintético, disposición para trabajar en grupos y equipos multidisciplinarios y capacidad de observación y abstracción, en un 13,11 y 9\%, respectivamente, según se observa en la figura número 11. 


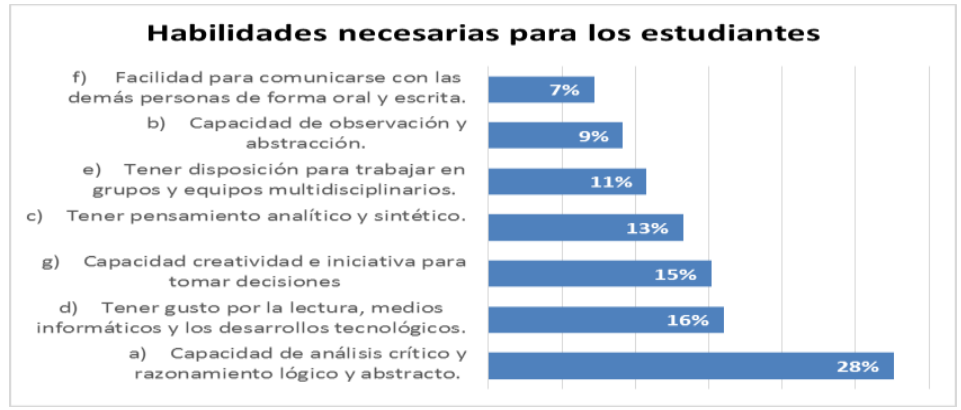

Figura 11. Habilidades del ingeniero de sistemas

Fuente: Elaboración propia

Con relación a las actitudes que se consideran indispensables para el estudiante de ingeniería de sistemas, los encuestados discurrieron que debe existir un interés por la tecnología, sus avances, sus problemas y descubrimientos en un 34\%, ser curioso y creativo en un 30\%, y el interés por la investigación y las matemáticas en un 17 y 19\%, respectivamente, según se ilustra en la figura número 12.

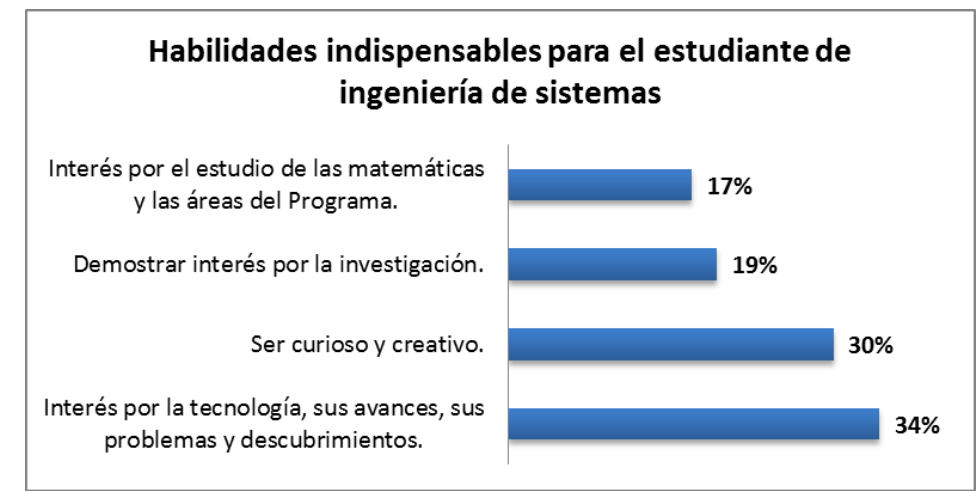

Figura 12. Actitudes del ingeniero de sistemas

Fuente: Elaboración Propia

Con relación al pensum o asignaturas del programa, los estudiantes opinaron en su mayoría que es muy completo, que las asignaturas se relacionan y complementan, que aportan en su proyecto de vida, genera nuevos conocimientos, competencias, desarrolla habilidades, prepara al mercado laboral y enseñan a desempeñarse. Con relación a lo que es más llamativo del programa, los encuestados contestaron en un 50\% que les gustaba el enfoque hacia la programación, y en un $13 \%$ los docentes, los horarios y la metodología respectivamente, en lo relacionado con la evaluación de la metodología de enseñanza recibida en el programa, el 73\% de los encuestados la consideraron buena, el $21 \%$ excelente y el $5 \%$ regular, como se enseña en la figura número 13 . 


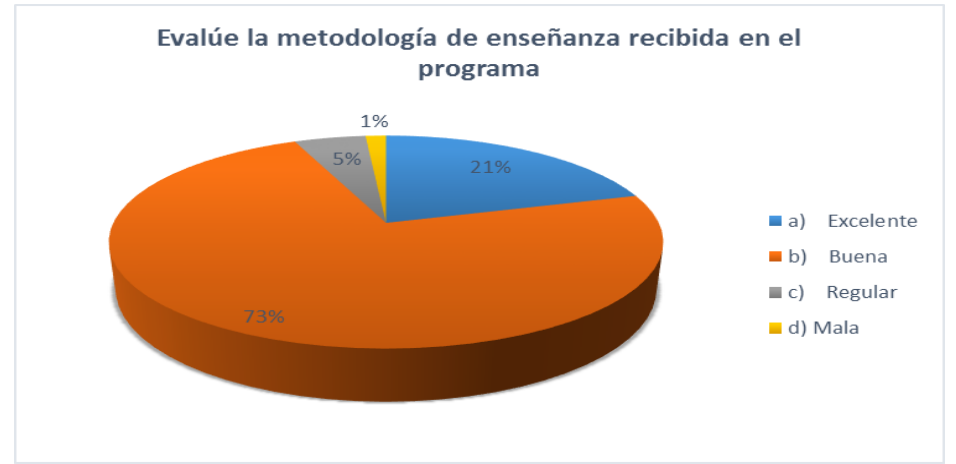

Figura 13. Evaluación de la metodología Fuente: Elaboración propia

De la infraestructura se puede observar que de los estudiantes, el $63 \%$ considera buena, el $20 \%$ regular y el $13 \%$ excelente, sin embargo el $30 \%$ de los encuestados manifestó inconformidad con la obsolescencia de los equipos, los equipos que estaban en mal estado, la falta de salas o tamaño de las mismas, la falta de velocidad en internet, entre otras como se muestra en la figura número 14.

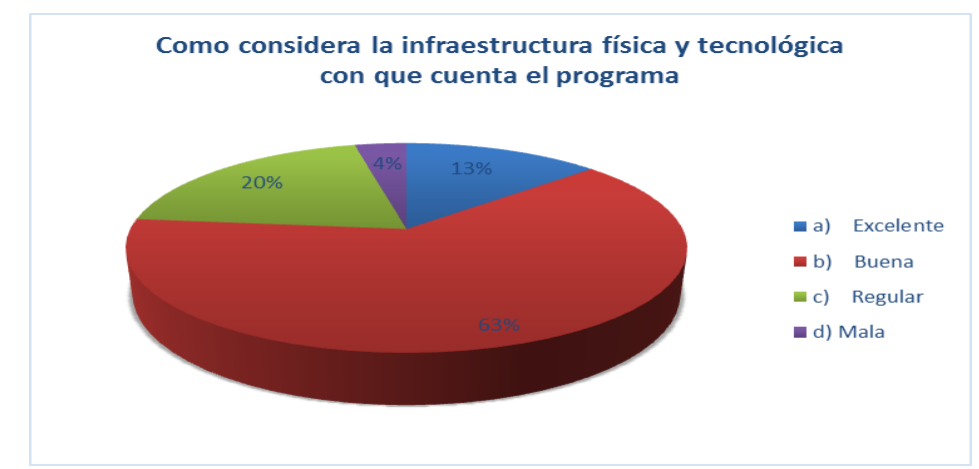

Figura 14. Evaluación de infraestructura Fuente: Elaboración propia

El 54\% de los encuestados consideran que el costo de la matrícula es razonable (universidad pública 1.75 SMLV. Universidad del Tolima) y (4 SMLV Universidad Privada. Universidad Cooperativa de Colombia), lo cual indica que el factor monetario no es concluyente al momento de tomar la decisión del tipo de educación recibida.

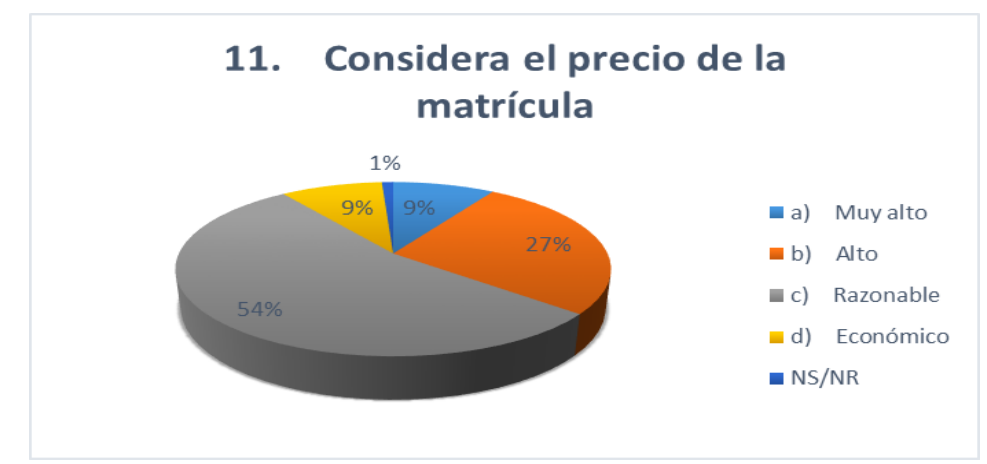

Figura 15. Precio en la matrícula

Fuente: Elaboración propia 


\section{Conclusiones}

Existe oferta suficiente de universidades que ofrecen el programa de Ingeniería de sistemas pero se debe incentivar la demanda, pues hay desconocimiento en el target. Los principales motivos de elección de carrera son aspectos subjetivos como gusto o afinidad, por lo cual se requiere sensibilizar a la población con relación a los beneficios del programa. Las universidades no están aplicando estrategias de marketing llamativas para los programas de ingeniería. Es muy importante tener en consideración diferentes aspectos de la población a quienes dirigen las estrategias de marketing. Existe interés del gobierno en incentivar la demanda, lo cual se puede canalizar a través de alianzas Universidad-Empresa- Estado.

\section{Recomendaciones}

Sostener una relación con los estudiantes para conocer sus expectativas y satisfacerlas. Crear vínculos entre la universidad y las instituciones de educación media y técnica, con el propósito de enseñar las verdaderas competencias de un ingeniero de sistemas. Buscar medios que fortalezcan y aumenten en los estudiantes de bachillerato el agrado por los sistemas. Crear e implementar estrategias que permitan el posicionamiento del programa. Conocer los gustos y expectativas del mercado meta, a fin de implementar acciones que fomenten la demanda del programa. Formular un plan de marketing, el cual promueva un análisis situacional que genere estrategias que permitan hacer más competitivo el programa de ingeniería objeto de estudio.

\section{Bibliografía}

Banco de la República. (2014). la educación actual en Colombia: situación actual y análisis de eficiencia. Borradores de economía, 2.

Chaquilla, S. (2 de Abril de 2013). mailxmail.com. Obtenido de Plan de mercadotecnia estrategias de marketing: http://www.mailxmail.com/

Eicher, J. C. (1988). Treinta años de Economía de la Educación. Ekonomiaz:. Revista vasca de economía, (12), 11-38.

Gibbs, A. (1997). "Focus Group". Obtenido de Social research update: http://sru.soc.surrey.ac.uk/SRU19.html

Hernández S, M. Á. (2014 de 02 de 10). Industria TIC colombiana necesita muchos más ingenieros. El Tiempo.

Hernández Sampieri, R., Fernández Collado, C., \& Baptista Lucio, P. (2010). Metología de la Investigación. Bogotá: McGraw Hill.

Hernandez, M. A. (10 de Febrero de 2014). Industria TIC colombiana necesita muchos más ingenieros. EL TIEMPO. 
INDEMER. (2010). Información de interes. Obtenido de Investigación y Estratégias de Marketing Educativo: http://www.colombiamercadeo.com

Marrón, M. A. (Octubre de 2014). Mg Soluciones. Obtenido de Reflexiones Sobre Posicionamiento: http://www.mgsolutions.es/pdf/posicionamiento.pdf

Muñiz, G. F. (16 de Octubre de 2014). Marketing XXI. Obtenido de Marketing en el siglo XXI: http://www.marketing-xxi.com/Marketing-siglo-xxi.html

Murray, R., \& Srephens, L. (2009). Probabilidad y estadística. México: McGraw-Hill.

Naranjo, H. C. (2011). Marketing educativo; desarrollo de una estrategia C.E.M. aplicado a la Universidad nacional de Colombia sede Manizales como base para la fidelización de clientes y complemento a la estrategia de C.R.M. Maniales: Tesis.

Philip, K., \& Armstrog, G. (2003). Fundamentos de Marketing. Pearson.

Rojas Villarce, J. Á. (4 de Noviembre de 2014). Técnicas no probabilísticas. Obtenido de http://tecnicas-noprobabilisticas.wikispaces.com/Jos\%C3\%A9+\%C3\%81ngel+Rojas+Villarce

Rojas, P., \& Maria, R. (2013). Rojas, P., \& Redondo, M Cómo preparar un plan de social media marketing. España: Grupo Planeta Spain.

Salas, V. M. (2002). Estimación de la rentabilidad de la inversión en educación universitaria de ciclo largo. España: Estadística Española.

Saldaña, E. J. (1999). Mercadotecnia para instituciones educativas. Contaduria y Administración.

Universidad Cooperativa de Colombia - Ingenieria de Sistemas. (2014). Proyecto Educativo del programa 2014- 2015. 15.

Universidad Cooperativa de Colombia. (18 de Agosto de 2013). Historia. Recuperado el 18 de agosto de 2014, de Historia: http://www.ucc.edu.co/institucion/Paginas/historia.aspx

Universidad de Antioquia. (7 de Octubre de 2014). Portal Universidad de Antioquia. Obtenido de http://www2.udea.edu.co/webmaster/indexudea.html

Universidad de Sonora. (4 de Noviembre de 2014). Departamento de matemáticas. Obtenido de http://www.estadistica.mat.uson.mx/Material/elmuestreo.pdf

Universidad del Valle. (2013). Estado de la educación superior en Iberamérica y en Colombia. El Observador Regional, 2.

Universidad Javeriana. (2001). Javesistemas.com. Obtenido de http://www.javesistemas.8m.com/encolombia/colombia.htm 